\title{
Acknowledgement to Reviewers of Marine Drugs in
} 2016

\author{
Marine Drugs Editorial Office \\ Published: 11 January 2017 \\ MDPI AG, St. Alban-Anlage 66, 4052 Basel, Switzerland; marinedrugs@mdpi.com
}

The editors of Marine Drugs would like to express their sincere gratitude to the following reviewers for assessing manuscripts in 2016.

We greatly appreciate the contribution of expert reviewers, which is crucial to the journal's editorial process. We aim to recognize reviewer contributions through several mechanisms, of which the annual publication of reviewer names is one. Reviewers receive a voucher entitling them to a discount on their next MDPI publication and can download a certificate of recognition directly from our submission system. Additionally, reviewers can sign up to the service Publons (https://publons.com) to receive recognition. Of course, in these initiatives we are careful not to compromise reviewer confidentiality. Many reviewers see their work as a voluntary and often unseen part of their role as researchers. We are grateful to the time reviewers donate to our journals and the contribution they make.

If you are interested in becoming a reviewer for Marine Drugs, see the link at the bottom of the webpage http://www.mdpi.com/reviewers.

The following reviewed for Marine Drugs in 2016:

Aarstad, Olav Andreas

Abe, Hideki

Afiyatullov, Shamil Sh.

Afonso, Clélia N.

Agyei, Dominic

Aiba, Tetsuya

Aki, Tsunehiro

Alber, Birgit E.

Al-Horani, Rami

Allen, Eric

Allsopp, Philip

Alo, Raffaella

Álvarez, Mercedes

Alves, Celso

Amsler, Charles

Anadon, Arturo

Anas, Andrea Roxanne

Andreadou, Ioanna

Andrianasolo, Eric

Anesi, Andrea

Anraku, Makoto

Arai, Ryoichi

Aranaz, Inmaculada

Aranda-Rodriguez, Rocio

Arduini, Alessandro
Arnason, John

Arnold, Eva

Avila, Conxita

Aviles, Francesc X.

Azuma, Kazuo

Azzimonti, Barbara

Bae, Hae-Rahn

Baker, Bill

Banack, Sandra

Banerjee, Monimoy

Banerjee, Pratik

Banskota, Arjun $\mathrm{H}$.

Baraka, Khaled

Barbosa, Mariana

Barnathan, Gilles

Barreira, Luísa

Basini, Giuseppina

Basti, Leila

Bates, Roderick W.

Beach, Daniel

Beaulieu, Lucie

Belas, Robert

Belovich, Joanne M.

Benkendorff, Kirsten

Berecki, Geza 
Berrue, Fabrice

Berry, John

Bianco, Armando Doriano

Bianco, I.D.

Bjørndal, Bodil

Blanco, Juan

Blandino, Giovanni

Blunt, John

Boateng, Joshua S.

Boddy, Christopher N.

Bøgwald, Jarl

Böhm, Volker

Bondon, Arnaud

Boquet, Didier

Bose, Utpal

Boussiba, Sammy

Boustie, Joel

Bovee, Toine F.H.

Brecker, Lothar

Breuss, Johannes

Brinkman, Diane

Brown, Lindsay

Brunel, Jean

Bugni, Tim

Bumgardner, Joel D

Burkhart, David J.

Cahoon, Lawrence

Calado, Ricardo

Caldwell, Gary

Callery, Patrick S.

Campos, Alexandre

Cañavate, José

Capon, Robert

CARAMELLA, CARLA

Carbone, Anna

Carmeli, Shmuel

Carrano, Lucia

Casapullo, Agostino

Caulier, Guillaume

Cerón-García, María Del Carmen

Cesaro, Attilio

Chang, Hsueh-Wei

Chang, Yi-tang

Chao, Louis Kuoping

Chauvierre, Cédric

Chen, Guoxun

Chen, Haixia

Chen, Jiann-Chu

Chen, Jih-Jung

Chen, Lihua

Chen, Shiu-Nan

Cheng, Yuan-Bin
Cherian, Philip

Cheung, Randy Chi Fai

Chiang, Meng-tsan

Chiesa, Giulia

Chi-Feng, Hung

Chinchilla, Rafael

Chiou, Ya-Ling

Chiu, Tsai-Hsin

Cho, Hyunah

Choi, Hyukjae

Choi, Jae Sue

Choi, Jong-il

Chong, Parkson Lee-gau

Chou, Joshua

Christensen, Lars

Chruszcz, Maksymilian

Chun, Byung-soo

Chynoweth, David P.

Ciavatta, M. Letizia

Cleary, Margot P.

Colliec-Jouault, Sylvia

Colombo, Diego

Constantino, Valeria

Costa, Monya

Costa, Pedro Reis

Costantini, Maria

Costantini, Susan

Coustau, Christine

Couzinet-mossion, Aurelie

Crespo, Mariano Sánchez

Cruz-Rivera, Edwin

Custódio, Luísa

D'Orazio, Nicolantonio

Dai, Mingji

Daly, Norelle

David, Betancur-ancona

De Agostini, Ariane

De Rosa, Maria Cristina

Defoirdt, Tom

Denny, Bill

Dharmaraj, Selvakumar

Di, Li

Dijkstra, Bauke W.

Dioum, Elhadji M.

Ducancel, Frédéric

Dufossé, Laurent

Duh, Chang-Yih

Duverger, Eric

Dyshlovoy, Sergey

Ebrahim, Hassan

Ebrahim, Weaam

Edirisinghe, Mohan 
Edrada-Ebel, Ruangelie

Egan, Suhelen

Ekuni, Daisuke

Elmore, Donald E.

Esteves, Ana Cristina

Evans, Franklin

Evin, Geneviève

Fam, Barbara

Fassouane, Aziz

Fatouros, Dimitrios

Faulkner, Simon

Feng, Yu

Fernández, José J.

Fernandez, Stefan

Fernando, Rodríguez-Serrano

Ferreira, Isabel

Ferrieres, Vincent

Festuccia, Claudio

Figueroa, Félix López

Fink-Gremmels, Johanna

Fitton, Helen

Fleury, Yannick

Foley, Sarah A.

Foubelo, Francisco

Franco, Chris

Frankenberg Dinkel, Nicole

Freitas, Ana

Freitas, Ana C.

Frolova, Liliya

Fujii, Ikuo

Fujii, Mikio

Fujii, Ritsuko

Fujita, Masaki

Fujita, Morifumi

Funane, Kazumi

Fuwa, Haruhiko

Gammone, MA

Garces, Esther

Garcia Camacho, F.

García-Mauriño, Sofía

Gavagnin, Margherita

Gavenonis, Jason

Genovese, Giuseppa

Ghoneum, M

Giannaccini, Martina

Gillis, Eric P.

Girgih, Abraham T.

Giunchedi, Paolo

Glaser, Keith

Glukhov, Alexey

Gnavi, Giorgio

Gnuzzo, Genoveffa
Gobe, Glenda C

Gomes, Nelson G.M.

Gozes, Illana

Gozzo, Franco

Gray, Christopher A.

Graziano, Guella

Green, Brad R.

Green, David W

Gribble, Gordon

Gribble, Gordon W.

Gross, Steven

Grothaus, Paul G.

Gsell, Alena

Guarnieri, Michael T.

Gueven, Nuri

Guillou, Hervé

Gushina, Irina

Gutiérrez, Marcelino

Gverzdys, Tomas

Häder, Donat P.

Hadwiger, Lee

Haertle, Thomas

Haglund, Peter

Hamann, Mark

Hampel, Daniela

Hansen, Espen

Hara, Hiroshi

Harder, Tilmann

Hardy, Joan

Hasegawa, Yasushi

Hatae, Noriyuki

Haug, Tor

Henriques, Sonia

Hernández-Ledesma, Blanca

Hernandez-Prieto, Miguel A.

Herraiz, T.

Hewage, Himaya Siddhihalu Wickrama

Hidari, Kazuya

Hildebrand, Mark

Hill, Robert A.

Hoffman, Angela M.

Honecker, Friedemann

Hopkinson, Brian

Hosokawa, Masashi

Hosokawa, Seijiro

$\mathrm{Hu}$, Huijuan

Huang, Chun-Yung

Huang, Tse-Hung

Hung, Andrew

Hwang, Pai-An

Hyun, Jin Won

Ianora, Adrianna 
Ibeanu, Gordon

Ibrahim, El-Sherbiny

Ibrahim, Hisham R.

Ibrahim, Salam

Indiveri, Cesare

Ioannou, Efstathia

Ishigami, Ken

Ishmael, Jane E.

Isnansetyo, Alim

Itabashi, Yutaka

Ito, Takuya

Izzotti, Alberto

J Martín-Rodríguez, Alberto

Jain, Mahendra Kumar

Jansen, Rolf

Jayakumar, Rangasamy

Jeon, You Jin

Jimbo, Mitsuru

Jin, Dong-Hoon

John, Ho Wing Shing

Johnson, John A

Joo, Hong-Gu

Jun, Joon-Young

Jung, Jee Hyung

Kaas, Quentin

Kalinin, Vladimir I

Kaludercic, Nina

Kanakkanthara, Arun

Kang, Hee

Kang, Heon-joong

Karagouni, Amalia D.

Karapanagiotidis, Ioannis T.

Kashman, Yoel

Katsifas, Efstathios A.

Kawai, Shigeyuki

Kawamura, Akira

Kerr, William

Khachik, Frederick

Khadka, Manoj

Khokhlatchev, Andrei

Khozin-Goldberg, Inna

Kidoaki, Satoru

Kijjoa, Anake

Kikuchi, Haruhisa

Kim, Duwoon

Kim, Leung

Kiss, Robert

Kitatani, Kazuyuki

Klettner, Alexa

Kobayashi, Toyoharu

Kogure, Noriyuki

Kolmar, Harald
Komaki, Hisayuki

Kong, In-soo

Kridel, Steven J.

Kuan, Yu-Hsiang

Kuepper, Frithjof

Kunanek, Julia

Kuo, Po-Lin

Kuo, Tzong-Fu

Kurihara, Hideyuki

Kurtán, Tibor

Kuyucak, Serdar

Kwak, Jong-Young

Kwon, Il-Keun

Kyzas, George

Laatsch, Hartmut

LaBarbera, Daniel V.

Labes, Antje

Lalk, Michael

Lang, Siegmund

Laparra, Moisés

Lau-Cam, Cesar A.

Lauder, Bob

Laurienzo, Paola

Lauritano, Chiara

Le Pogam, Pierre

Leão, Pedro N.

Lee, Bong Ho

Lee, Che-Hsin

Lee, Chia-hung

Lee, Ji-young

Lee, Jun Sik

Lee, Kyubock

Lee, Tsung-Han

Lee, Victor

Lee, Yeon-Ju

Leone, Antonella

Li, Ning

Li, Yan

Liakos, Ioannis L.

Liang, Yu-chih

Liaw, Chih-Chuang

Licea Navaro, Alexei Fedorovish

Lin, Hai-shu

Lin, Hsi-hsien

Lin, Zhenjian

Liou, Chian-Jiun

Little, R. Dan

Liu, Junying

Liu, Pinghua

Lo Giudice, Angelina Lo

Lopanik, Nicole B.

Louzao, M. Carmen 
Lu, Jun

Lu, Mei-Chin

$\mathrm{Lu}$, Sizhao

Lüdeke, Steffen

Luesch, Hendrik

Lynch, Jennifer

M, Valan Arasu

M. Greene, Catherine

MacMillan, John

Maderna, Andreas

Madhukar, Burra

Maeda, Hayato

Majumder, Kaustav

Mancini, Ines

Manganiello, Gelsomina

Mangoni, Alfonso

Marino, Angela

Martin, Jessica

Martin, Luc J.

Martínez-Alvarez, Oscar

Marx, Florentine

Mass, Tali

Matsunaga, Shigeki

Matsuura, Hiroshi

Maupin, C. Mark

Mazur-Marzec, Hanna

McCall, Jennifer R.

McCormick, Rachel

McMullin, David R.

Meadus, William Jon

Mebs, Dietrich

Medina, Isabel

Menna, Marialuisa

Metcalf, James

MEUNIER, Bernard

Meyer, Anne S

Miert, Sabine Van

Milthorpe, Bruce

Mishra, Nigam

Miyaoka, Hiroaki

Miyashita, Kazuo

Miyazaki, Yasunori

Mizuno, Masashi

Moffit, Michelle C.

Mohammad, Altaf

Mölleken, Helga

Monti, Maria Chiara

Morabito, Marina

Morales, Diana

Moran, Yehu

Moreno, Antonio D.

Morfini, Gerardo
Mori, Naoki

Morimura, Shigeru

Mossialos, Dimitris

Motti, Cherie

Müller, Werner

Müller, Werner E.G.

Mundt, Sabine

Muramoto, Koji

Murch, Susan

Murphree, S. Shaun

Murphy, Patrick J.

Muzzarelli, Riccardo A. A.

Naderer, Thomas

Nánási, Péter

Nasopoulou, Constantina

Nasri, Rim

Navarro, Juan Carlos

Nazar, Hamde

Nelson, David

Newman, David

Nicoletti, Rosario

Nishimura, Shinichi

Nisticò, Roberto

Noh, Jeong Sook

Norton, Raymond

Novakovic, Katarina

Nunes, Cláudia

O'Doherty, George

Oh, Dong-Chan

Oh, Jung Hwan

Okunade, Adewole L.

Ortiz, Abel

Osaki, Tomohiro

Osborn, Helen

Ozeki, Yasuhiro

Pae, Ae Nim

Pan, Cheol-ho

Pattenden, Gerald

Paulsen, Berit Smestad

Pautz, Andrea

Pavon-Djavid, Graciela

Peigneur, Steve

Pereira, José Augusto

Pereira, Leonel

Perez, Maria Jose

Perez-Jimenez, Jara

Perillo, Emiliana

Pescitelli, Gennaro

Petit, Karina

Petkuviene, Jolita

Picot, L.

Piganelli, Jon 
Piggott, Andrew

Pillay, Viness

Pinheiro, Maria Lúcia Belém

Pinto, Diana C. G. A.

Plaza, Alberto

Pleissner, Daniel

Pouchus, Yves-Francois

Proksch, Peter

Pucciarelli, Sandra

Qaralleh, Haitham N.

Qiu, Huan

Quesada, Antonio

Quinn, Mark T.

Quinn, Ron

Qvit, Nir

Raabe, Gerhard

Raatz, Susan K.

Radovic, Jagos R.

Rafael, Diana

Rahman, Azizur

Raina, Jean-Baptiste

Rämä, Teppo

Ramadan, Usama

Ramos, Adrián M.

Rao, Christopher

Raposo, Maria Filomena Jesus

Rasenick, Mark

Rebollo, Angelita

Rebuffat, Sylvie

Rein, Kathleen

Reitzel, Adam

Reynisson, Jóhannes

Rho, Jung-Rae

Rinaudo, Marguerite

Ringe, Dagmar

RIzzo, Angela Maria

Robertson, Andrew

Roh, Changhyun

Roh, Seong-Soo

Rokstad, Anne Mari

Romano, Giovanna

Rosen, Johan

Roullier, Catherine

Roussis, Vasileios

Rudd, David

Ruiz-Rodríguez, Magdalena

Russell, Fraser D.

Russo, Gian Luigi

Rustad, Turid

Ryu, Bomi

Sabatier, Jean-Marc

Sadamoto, Hisayo
Sakai, Ryuichi

Salanoubat, Marcel

Santos Costa De Morais, Rui Manuel

Santos, Abel

Santos, Susana

Sarojini, Vijayalekshmi

Sass, Henrik

Sasso, Severin

Sato, Kenji

Satomi, Yoshiko

Schenk, Peer M.

Schmalz, Hans-Guenther

Schroeckh, Volker

Schroeder, Christina I.

Sebestik, Jaroslav

Senatore, Adriano

Seo, Jung-Kil

Shaw, Lindsey

Shenawy, Nahla S. El

Shepherd, Suzanne M.

Sheu, Jyh-Horng

Shin, Hee

Shin, Jongheon

Shindo, Kazutoshi

Sidorenko, Alexander

Sieiro, Carmen

Sigurjonsson, Olafur E.

Silva, Artur

Sim, Sang Jun

Simpson, Tom

Smith, Alan

Söderhäll, Irene

Solaiman, Daniel K.Y.

Sosa, Silvio

Soueidan, Assem

Spahn, Viola

Sreedasyam, Avinash

Stoskopf, Michael

Sugahara, Takuya

Suleria, Hafiz Ansar Rasul

Sumi, Shoichiro

Sung, H.H.

Sung, Ping-Jyun

Sutherland, John B.

Sutherland, Mark

Suzuki, Iwane

Tadahiro, Suzuki

Tahul, Maria B Badia

Takahashi, Daisuke

Takahashi, Koretaro

Takaichi, Shinichi

Tanaka, Tsuyoshi 
Tarman, Kustiariyah

Tartaglione, Luciana

Tepe, Jetze J.

Teta, Roberta

Thomas, François

Thomas, Jim

Thomas, Olivier P.

Thoo-Lin, Paul Kong

Thurber, Greg M.

Tokuda, Masaharu

Tommonaro, Giuseppina

Tomoda, Hiroshi

Topakas, Evangelos

Trincone, Antonio

Tsodikov, Oleg

Tsuda, Masashi

Turk, Tom

Tutino, Maria Luisa

Ubhayasekera, Sarojini J. K. A.

Ulanova, Dana

Umeyama, Akemi

Ustyuzhanina, Nadezhda E

Vale, Paulo

Valentao, Patrícia

Valério, Elisabete

Van Berkel, Willem

Van Lanen, Steven

Vapaatalo, Heikki

Varela-Álvarez, Elena

Varese, Giovanna Cristina

Vázquez, J. A.

Veiga, María-Dolores

Vieira, Helena

Vílchez, Carlos

Vogt, Miriam Annika

Voigt, Oliver

Von Schacky, Clemens

Voorspoels, Stefan

Wang, Changyun

Wang, Cuihua
Wang, Piwen

Wang, Qun

Wang, San-Lang

Wang, Shang-kwei

Wang, Tzu-Wei

Wang, Wei

Wang, Wei-Kuang

Wang, Wei-Lung

Wietz, Matthias

Wioleta, Kowalczyk

Witczak, Zbigniew

Witulski, Bernhard

Wlodawer, Alexander

$\mathrm{Wu}$, Chang-Jer

$\mathrm{Wu}$, Chieh-Hsi

$\mathrm{Wu}$, Chin-Chung

Wu, Hongfu

Yamada, Shuhei

Yamada, Sohsuke

Yamada, Takeshi

Yamashita, Mari Yotsu

Yamazaki, Hiroyuki

Yan, Ning

Yang, Jyisy

Yarotskyy, Viktor

Young, Howard

Young, Wayne

Younis, Rather

Yoza, Brandon A.

Yuan, Zhihong

Yue, PatrickYingKit

Zaferanloo, Bita

Zaharof, David

Zhang, Fan

Zhang, Fuming

Zhang, Wen

Zhang, Yifan

Zhou, Yu-Dong

Zhu, Mike

Zubía, Eva

(c) 2017 by the authors; licensee MDPI, Basel, Switzerland. This article is an open access article distributed under the terms and conditions of the Creative Commons Attribution (CC-BY) license (http://creativecommons.org/licenses/by/4.0/). 